\title{
Professional self-regulation: learning from the disciplinary process
}

\author{
Robert J. Byrick, MD
}

Received: 27 June 2013/Accepted: 18 July 2013/Published online: 2 August 2013

(C) Canadian Anesthesiologists' Society 2013

Medical regulatory colleges have a mandate to protect and promote the health and safety of the public by regulating the practice of medicine by physicians. In Canada, provincial colleges of physicians and surgeons fulfill this mandate through activities related to a) the registration of physicians to practice, $b$ ) the regulation of how physicians practice (either proactive or reactive regulatory activities), and c) remediation of physicians where specific deficiencies are identified and where remediation is appropriate.

Throughout most of the 150-year history of selfregulation of the medical profession in Canada, this role has been performed primarily by reactive regulation, i.e., disciplining physicians who have been found to be practicing in a manner that members of the profession would consider to be dishonourable, disgraceful, or unprofessional. As important as remediation may be, the disciplinary process remains a key means of maintaining the public trust in the profession. This is reactive regulation, as it results from a specific complaint, inquiry, or report to the college about a physician's behaviour or performance. It is important to emphasize that most colleges have remediation programs that manage clinical practice deficiencies identified during the

The author is a member of Council of the College of Physicians and Surgeons of Ontario and has no other competing interests to declare.

L'auteur est membre du Conseil de l'Ordre des médecins et chirurgiens de l'Ontario et n'a pas d'autre conflit d'intérêt à déclarer.

R. J. Byrick, MD ( $₫)$

Department of Anesthesia, St. Michael's Hospital, University of

Toronto, 30 Bond Street, Toronto, ON M5B 1W8, Canada

e-mail: robert.byrick@utoronto.ca complaint process. Disciplinary procedures are invoked only when major unprofessional practices are found.

By law, the college's disciplinary proceedings and outcomes are transparent to the public. Disciplinary findings result from a formal adversarial legal process after an extensive investigation of the merits of the allegations. Formal charges are filed, and the physician involved is allowed to mount a thorough defense to the allegations. Although individual disciplinary findings are published by each provincial regulatory body, the report presented by Alam et al. in this issue of the Journal ${ }^{1}$ is the first systematic evaluation of disciplinary findings against anesthesiologists in Canada. The report follows the publication of their data on all disciplined physicians in Canada from 2000-2009. ${ }^{2}$ The authors conclude that publication of the causes of disciplinary actions may result in "interventions aimed at educating physicians around standard of care" and prevent such problems. Whether education can prevent such serious lapses of professionalism remains speculative at best, but their data give us the first specialty-specific, national perspective on this important issue.

Their first contribution is to compare the scope of the problem for anesthesiologists with that for all physicians in Canada. They emphasize that there were 721 disciplinary findings against physicians of all specialties in Canada from 2000-2011, whereas only 11 findings were against anesthesiologists. This frequency represents disproportionately fewer anesthesiologists than many other specialties. This result differs from the report from California's State Medical Board where anesthesiologists were overrepresented among disciplined physicians. ${ }^{3}$

Complaints that reach the Discipline Committee represent only $2-3 \%$ of the total number of patient complaints about physicians. This number probably 
underestimates the incidence of physicians who would be found to be unprofessional, as some surrender their license voluntarily when allegations of unprofessional conduct are made. Their resignation limits the investigative process at that point. We also do not know the denominator for the number of complaints to regulators about anesthesiologists in Canada during this time frame. Although there were only eleven disciplinary findings, there were certainly many more reports of concern about anesthesiologists to regulatory colleges. Most (97-98\%) patient complaints, coroners' reports, and hospital inquiries result in dispositions that are not referrals to the Discipline Committee. One limitation of using this public database of disciplinary findings is that various regulatory colleges may have different thresholds for referral to discipline, creating a "selection bias". There is no doubt that disciplinary findings represent the "tip of the iceberg" albeit an important tip - when it comes to patient complaints and the public's concerns and perceptions of our care.

The authors ${ }^{1}$ describe general features of the cases that result in disciplinary findings for our specialty. There were some similarities between these data for anesthesiologists and their data for the profession as a whole. ${ }^{2}$ For example, all disciplined anesthesiologists were male. The most common causes for discipline were standard of care issues, inappropriate prescribing, and fraudulent behaviour. These are significant and important areas of professional practice that should be included in designing educational programs for practicing anesthesiologists. We are not given the details for these specific cases; however, they correctly emphasize that the nature and scope of clinical practice influences the frequency of disciplinary findings. For example, anesthesiologists practicing in chronic pain medicine need to have a heightened concern about inappropriately prescribing opiates.

The authors reported that only one anesthesiologist was disciplined for self-use of drugs and alcohol. ${ }^{1}$ This is undoubtedly a significant underestimation of the incidence, and there is no room for complacency regarding the issue of substance abuse in our specialty. The authors correctly emphasize that substance abuse problems and the rehabilitation process is usually managed confidentially by the provincial physician health program with the approval of the regulatory college, not through the disciplinary process. For anesthesiologists, this problem remains an important issue and emphasizes that data on disciplinary findings have limitations with respect to generalized usefulness to prioritize educational programs.

It is clear that disciplinary findings do not represent a balanced picture of the complaints received from patients or concerns relating to standard of care. Bismark et al. ${ }^{4}$ recently reported a study of patient complaints to regulators in Australia. The reporting of all complaints to the regulator would give a more balanced assessment of patient concerns than disciplinary findings. In the United Kingdom, Campbell et al. ${ }^{5}$ recently analyzed all complaint cases referred to the General Medical Council (GMC) concerning anesthesiologists in 2009. Their data showed that anesthesiologists had a lower rate of referral compared with doctors in general $(P<0.0001)$. There are differences between countries regarding how patient complaints are managed by the regulatory authorities, so comparisons are difficult. Nevertheless, interesting patterns emerge. Campbell et al. ${ }^{5}$ found that higher rates of referral to the GMC were made for older doctors, males, and those who qualified outside the United Kingdom. It was not infrequent for referred anesthesiologists to have more than one allegation of unprofessional conduct against him/her (225 allegations against 81 anesthesiologists). This latter finding is similar to the Australian data. ${ }^{4}$ Interestingly, Alam et al. found that one of the nine Canadian anesthesiologists had three disciplinary findings. ${ }^{1}$ The authors recognize the need to identify physicians with professionalism problems early in their careers and to take remedial action to prevent further unprofessional conduct and patient harm.

Alam et al. ${ }^{1}$ point out two important findings that require specific comment in the Canadian context. First, the mean number of years in practice of anesthesiologists who have disciplinary findings (31.9) is similar to all physicians as a group (29.2). Although we do not know the exact age of the anesthesiologists who had disciplinary findings, it is obvious that older physicians are overrepresented for both anesthesiologists and the medical profession in general. This has been reported in other analyses of disciplinary findings and complaints. ${ }^{3,5}$ Care should be taken when interpreting Alam et al.'s data, as the number of anesthesiologists is small $(n=9)$. Nevertheless, there is a growing body of evidence suggesting that age is an independent risk factor for substandard clinical performance. $^{4}$ It should be emphasized that other contributing factors identified include poor performance in medical school, solo practice, lack of hospital privileges, lack of specialty qualifications, practicing outside scope of training, high clinical volumes, fatigue, stress and burnout, as well as health issues. ${ }^{6}$ Medical professionalism in all specialties demands that we address the issue of age and maintenance of competence. Regulators are increasingly targeting age-based screening of physicians for competency assessment. Then again, there are few studies that analyse the effectiveness of either peer assessments of aging physicians or subsequent structured clinical educational and remediation programs for improving clinical performance. It is essential that we take the lead in this issue and develop relevant assessment tools and remediation strategies before either the public demands it or another organization undertakes the task. 
Second, Alam et al. point out the prevalence of international medical graduates (IMGs) among anesthesiologists who were subjects of disciplinary findings. ${ }^{1}$ They found that two-thirds of anesthesiologists who were disciplined were IMGs, yet in their other study, only one-third of all physicians were IMGs. ${ }^{2}$ There is no statistically significant difference between these rates, and care is needed in the interpretation. We do not know any details of the background education of the IMGs in Alam et al.'s study, and many may have completed their specialty training in Canada. It is important to emphasize that there was no difference between the incidence of discipline among IMGs $v s$ a control group in the California study. ${ }^{3}$ The sample size is too small $(n=9)$ for any statistically valid analysis, and no conclusions should be made from these data.

In conclusion, this is the first report of disciplinary findings concerning anesthesiologists by Canadian regulatory colleges. These data are reassuring in that the total frequency of disciplinary findings against anesthesiologists is lower than in the medical profession as a whole. Medical regulatory colleges are responsible for certifying the professional characteristics of physicians and thus have an obligation to ensure that professional misconduct, when identified, is dealt with appropriately. In spite of the many limitations of using disciplinary findings as a measure of the incidence of substandard professional care and as a guide to educational programs, Alam et al. provide insight into the process and suggest that college-derived data can be useful information for specific specialties. ${ }^{1}$ The regulatory colleges are limited by confidentiality in much of their work; on the other hand, by working in concert with specialty societies and educational bodies, the regulators have the potential to provide valuable information that could direct educational programs to areas of need. Such collaboration between the regulator and the regulated profession can serve the patients whom we aim to protect as well as preserve the public trust in our profession.

\section{L'autoréglementation professionnelle: quelques leçons à tirer des procédures disciplinaires}

Le mandat des organismes de réglementation médicale est de protéger et de promouvoir la santé et la sécurité du public en régissant la pratique de la médecine par des médecins. Au Canada, les ordres provinciaux de médecins et chirurgiens remplissent ce mandat par le biais d'activités liées aux domaines suivants: a) l'autorisation de pratiquer des médecins; b) la réglementation de la façon dont les médecins pratiquent (via des activités réglementaires proactives ou réactives), et c) les mesures de remédiation envers les médecins lorsque des lacunes spécifiques sont identifiées et lorsque de telles mesures sont adaptées.

Durant les 150 ans d'histoire de l'autoréglementation de la profession médicale au Canada, cette dernière mission a été accomplie principalement en réglementant de façon réactive, c'est-à-dire en imposant des mesures disciplinaires aux médecins dont la pratique aurait été jugée peu honorable, honteuse ou non professionnelle par les membres de la profession. Bien que la remédiation soit cruciale, la procédure disciplinaire demeure une façon clé de maintenir la confiance du public en la profession. Il s'agit là d'une réglementation réactive, étant donné qu'elle découle d'une plainte, d'une enquête ou d'un compte-rendu spécifique transmis à l'ordre et déplorant le comportement ou la performance d'un médecin. Il est important de souligner que la plupart des ordres ont des programmes de remédiation en place, lesquels ont pour objectif de pallier les lacunes de la pratique clinique identifiées au cours du processus de plainte. La procédure disciplinaire n'est invoquée que lorsqu'il y a pratique non professionnelle majeure.

De par la loi, les actions disciplinaires d'un ordre professionnel et leurs résultats sont transparents pour le public. Les jugements disciplinaires sont le résultat d'un processus légal accusatoire formel à la suite d'une enquête approfondie sur le bien-fondé d'allégations. Des accusations formelles sont émises, et le médecin impliqué est autorisé à préparer une défense complète contre ces allégations. Bien que les jugements disciplinaires individuels soient publiés par chaque organisme provincial de réglementation, le compte-rendu d'Alam et coll., publié dans ce numéro du Journal, constitue la première évaluation méthodique des jugements disciplinaires à l'endroit d'anesthésiologistes au Canada. Ce rapport suit la publication d'un autre s'intéressant à tous les médecins ayant fait l'objet de mesures disciplinaires au Canada entre 2000 et $2009 .^{2}$ Les auteurs concluent que la publication des causes de mesures disciplinaires pourrait favoriser la création «d'interventions destinées à former les médecins concernant les normes de soins » et prévenir de tels problèmes. Il reste à prouver que la formation puisse véritablement prévenir de tels écarts de conduite professionnelle; toutefois, les résultats d'Alam et coll. constituent la première perspective nationale spécifique à notre spécialité sur cette question cruciale.

La première étape de l'étude a été de comparer l'étendue du problème chez les anesthésiologistes par rapport à tous les médecins au Canada. Les auteurs rapportent qu'il y a eu 721 jugements disciplinaires portés à l'endroit de 
médecins, toutes spécialités confondues, au Canada entre 2000 et 2011, alors que seuls onze de ces jugements impliquaient des anesthésiologistes. Toutes proportions gardées, le nombre d'anesthésiologistes impliqués est faible comparativement à bien d'autres spécialités. Ce résultat est très différent du rapport publié par le Conseil médical de l'État de Californie, dans lequel les anesthésiologistes étaient surreprésentés parmi les médecins ayant fait l'objet de mesures disciplinaires.

Les plaintes qui se rendent jusqu'au Comité de discipline ne représentent que 2-3\% du nombre total de plaintes de patients à l'endroit de médecins. Ce nombre sous-estime probablement l'incidence de médecins dont le comportement serait jugé non professionnel, étant donné que certains consentent à renoncer à leur permis de pratique lorsque des allégations de conduite non professionnelle sont portées. Leur démission met alors un terme au processus d'enquête. Nous ne connaissons pas non plus le dénominateur du nombre de plaintes faites aux organismes de réglementation concernant les anesthésiologistes au Canada au cours de cette période. Bien qu'il n'y ait eu que onze jugements disciplinaires, il y a indubitablement eu bien plus de rapports transmis aux organismes de réglementation faisant état d'inquiétudes et de préoccupations concernant le comportement de certains anesthésiologistes. La plupart (97-98\%) des plaintes de patients, des rapports de coroners et des enquêtes hospitalières se concluent par des arrangements qui ne nécessitent pas l'implication du Comité de discipline. En outre, l'une des limites à l'utilisation d'une telle base de données publique de jugements disciplinaires est que les divers organismes de réglementation pourraient avoir différents seuils avant de passer le relais au Comité de discipline, créant ainsi un «biais de sélection ». Il ne fait aucun doute que les jugements disciplinaires ne représentent que «la pointe de l'iceberg »- même si cette pointe est importante - quand il s'agit des plaintes des patients et des inquiétudes et perceptions de la communauté concernant les soins que nous prodiguons.

Les auteurs ${ }^{1}$ décrivent des caractéristiques générales des cas entraînant des jugements disciplinaires dans notre spécialité. On peut observer certaines similarités entre les données concernant les anesthésiologistes et celles concernant la profession médicale en général. ${ }^{2}$ Par exemple, tous les anesthésiologistes ayant fait l'objet de mesures disciplinaires étaient des hommes. Les causes les plus fréquentes ayant mené à des mesures disciplinaires étaient liées à des questions de normes de soins, de prescriptions inadaptées et de comportement frauduleux. Il s'agit là de champs importants de notre pratique professionnelle qui doivent être inclus dans la conception de programmes de formation pour les anesthésiologistes en pratique. Les auteurs ne donnent pas les détails de ces cas en particulier; toutefois, ils soulignent à point nommé que la nature et l'étendue de la pratique clinique influencent la fréquence des jugements disciplinaires. Par exemple, les anesthésiologistes pratiquant en médecine de la douleur chronique doivent être particulièrement prudents lorsque vient le moment de prescrire des opiacés afin de ne pas le faire de façon inadaptée.

Les auteurs rapportent qu'un seul anesthésiologiste a fait l'objet de mesures disciplinaires pour avoir lui-même utilisé des médicaments et de l'alcool. ${ }^{1}$ Il s'agit indubitablement d'une sous-estimation considérable de l'incidence de ce problème, et la complaisance n'a aucune place lorsqu'il est question d'abus de substances dans notre spécialité. Les auteurs insistent à juste titre sur le fait que les problèmes d'abus de substances et le processus de réhabilitation sont en général gérés de façon confidentielle par le programme provincial pour la santé des médecins avec l'accord de l'organisme de réglementation, et non via un processus disciplinaire. Chez les anesthésiologistes, ce problème demeure important. Cet exemple démontre que les données portant sur les jugements disciplinaires ont leurs limites quand il s'agit d'établir des priorités dans les programmes de formation.

Il est clair que les jugements disciplinaires ne sont pas le reflet exact des plaintes reçues de patients ou des inquiétudes liées aux normes de soins. Bismark et coll. ${ }^{4}$ ont récemment publié une étude portant sur les plaintes des patients transmises aux organismes de réglementation en Australie. La communication de toutes les plaintes à l'organisme de réglementation permettrait d'obtenir une évaluation plus équilibrée des préoccupations des patients que les jugements disciplinaires. Au Royaume-Uni, Campbell et coll. ${ }^{5}$ ont récemment analysé tous les cas de plaintes référées au Conseil médical général (CMG) concernant les anesthésiologistes en 2009. Leurs données ont montré que le taux de plaintes était plus bas à l'endroit des anesthésiologistes qu'à l'endroit des médecins en général $(P<0,0001)$. Chaque pays a sa propre façon de gérer les plaintes des patients via les organismes de réglementation. Dès lors, les comparaisons sont difficiles. Ceci étant dit, certaines tendances intéressantes apparaissent. Campbell et coll. ${ }^{5}$ ont observé que des taux plus élevés de plaintes ont été référées au CMG à l'endroit de médecins plus âgés, de sexe masculin, et de ceux formés hors du Royaume-Uni. Il était fréquent que les anesthésiologistes contre lesquels une plainte avait été déposée aient plus d'une allégation de comportement non professionnel à leur endroit (225 allégations contre 81 anesthésiologistes). Cette dernière observation se retrouve également dans l'étude australienne. ${ }^{4}$ Fait intéressant, Alam et coll. ont observé que l'un des neuf anesthésiologistes canadiens faisait l'objet de trois 
jugements disciplinaires. ${ }^{1}$ Les auteurs conviennent qu'il faut identifier les médecins présentant des problèmes de professionnalisme tôt dans leur carrière et mettre en place des mesures correctives afin de prévenir tout comportement non professionnel futur et tout tort aux patients.

Alam et coll. ${ }^{1}$ présentent deux observations importantes qui nécessitent une analyse plus poussée dans le contexte canadien. En premier lieu, le nombre moyen d'années de pratique des anesthésiologistes qui font l'objet de jugements disciplinaires $(31,9)$ est semblable à celui de tous les médecins en tant que groupe $(29,2)$. Bien que nous ne connaissions pas l'âge exact des anesthésiologistes ayant fait l'objet de jugements disciplinaires, il est évident que les médecins plus âgés sont surreprésentés dans le groupe des anesthésiologistes et dans celui de la profession médicale en général. Cette observation a été rapportée dans d'autres analyses de jugements disciplinaires et de plaintes. $^{3,5}$ Il convient de faire attention lors de l'interprétation des données d'Alam et coll., étant donné que le nombre d'anesthésiologistes est petit $(n=9)$. Toutefois, les données probantes suggérant que l'âge est un facteur de risque indépendant de performance clinique de qualité inférieure augmentent. ${ }^{4}$ Il convient de souligner qu'il existe d'autres facteurs identifiés comme contribuant à ce problème, notamment: de mauvais résultats pendant la formation en médecine, la pratique en solo, l'absence de privilèges hospitaliers, le manque de qualifications de spécialité, la pratique hors du domaine de formation, une charge clinique élevée, la fatigue, le stress et l'épuisement professionnel, ainsi que les problèmes de santé. ${ }^{6}$ Dans toutes les spécialités, le professionnalisme médical requiert que nous nous attaquions à la question de l'âge et du maintien des compétences. Les organismes de réglementation ciblent de plus en plus un dépistage fondé sur l'âge des médecins pour évaluer leurs compétences. Cependant, il existe peu d'études analysant l'efficacité de l'évaluation par leurs pairs des médecins vieillissants ou les programmes de formation et de remédiation cliniques structurés subséquents pour améliorer la performance clinique. Il est crucial que nous prenions le taureau par les cornes et mettions au point des outils d'évaluation pertinents ainsi que des stratégies de remédiation - avant que le public ne l'exige ou qu'un autre organisme ne s'attelle à la tâche.

Deuxièmement, Alam et coll. soulignent la prévalence de diplômés hors du Canada et des États-Unis parmi les anesthésiologistes qui ont fait l'objet de jugements disciplinaires. ${ }^{1}$ Ils observent que deux tiers des anesthésiologistes ayant fait l'objet de tels jugements étaient des diplômés hors Canada et États-Unis; toutefois, dans leur autre étude, seul un tiers de tous les médecins avait étudié ailleurs. ${ }^{2}$ Il n'existe pas de différence significative d'un point de vue statistique entre ces taux, et il convient d'être prudent dans leur interprétation. Nous ne connaissons pas les détails concernant la formation de base de ces diplômés dans l'étude d'Alam et coll., et bon nombre auraient tout à fait pu faire leur spécialisation au Canada. Il est important de souligner qu'il n'y a eu aucune différence entre l'incidence de mesures disciplinaires parmi les diplômés provenant de l'étranger et un groupe témoin dans l'étude californienne. ${ }^{3}$ La taille d'échantillon est trop petite $(n=9)$ pour toute analyse statistique valable, et aucune conclusion ne devrait être tirée de ces données.

En conclusion, il s'agit du premier compte-rendu portant sur les jugements disciplinaires à l'endroit d'anesthésiologistes émis par les organismes de réglementation canadiens. Ces données sont rassurantes en ce que la fréquence totale des jugements disciplinaires à l'endroit d'anesthésiologistes est plus basse que dans la profession médicale dans son intégralité. Les organismes de réglementation médicale sont responsables de l'accréditation des caractéristiques professionnelles des médecins et ont dès lors l'obligation d'assurer que la mauvaise conduite professionnelle est rapidement et adéquatement prise en charge lorsqu'elle est identifiée. En dépit des nombreuses limites liées à l'utilisation de jugements disciplinaires comme mesure de l'incidence des soins professionnels en-deçà de la norme et comme guide à l'élaboration de programmes de formation, Alam et coll. ouvrent tout de même une fenêtre sur ce processus et établissent que des données provenant des ordres professionnels peuvent constituer des informations utiles pour des spécialités en particulier. ${ }^{1}$ Les organismes de réglementation sont tenus à la confidentialité dans la grande partie de leur travail; ceci étant dit, en travaillant de concert avec les sociétés des spécialités et les organismes de formation, ces organismes ont la possibilité de fournir des informations précieuses qui pourraient orienter les programmes de formation vers les domaines où la formation est nécessaire. Une telle collaboration entre les organismes de réglementation et la profession régie pourrait bénéficier aux patients que nous visons à protéger et nous aider à préserver la confiance du public en notre profession.

\section{References}

1. Alam A, Khan J, Liu J, Klemensberg J. Griesman J, Bell CM. Characteristics and rates of disciplinary findings amongst anesthesiologists by professional colleges in Canada. Can $\mathrm{J}$ Anesth 2013: 60: this issue. DOI:10.1007/s12630-013-0006-8.

2. Alam A, Klemensberg J, Griesman J, Bell CM. The characteristics of physicians disciplined by professional colleges in Canada. Open Med 2011; 5: e166-72.

3. Morrison J, Wickersham P. Physicians disciplined by a state medical board. JAMA 1998; 279: 1889-93. 
4. Bismark MM, Spittal MJ, Gurrin LC, Ward M, Studdert DM. Identification of doctors at risk of recurrent complaints: a national study of healthcare complaints in Australia. BMJ Qual Saf 2013; 22: $532-40$.

5. Campbell G, Rollin AM, Smith AF. Cases relating to anaesthetists handled by the UK General Medical Council in 2009: methodological approach and patterns of referral. Anaesthesia 2013; 68: 453-60.

6. Moutier CY, Bazzo DE, Norcross WA. Approaching the issue of the aging physician population. Journal of Medical Regulation 2013; 99: $10-8$. 